\title{
Lamiaceae endémicas del Perú
}

\author{
Maritza Rodriguez
}

Paz Soldán 316, Miraflores. Arequipa, Perú.

maritza_gladys@yahoo.es

\section{Resumen}

La familia Lamiaceae es reconocida en el Perú por presentar alrededor de 21 géneros y 190 especies ((Brako \& Zarucchi, 1993; Ulloa Ulloa et al., 2004), mayormente hierbas y arbustos. En este trabajo reconocemos 57 especies endémicas en nueve géneros. El género Salvia es el más rico en especies endémicas. Las especies de Lamiaceae endémicas ocupan principalmente las regiones Mesoandina y Bosques Muy Húmedos Montanos, entre los 1500 y $4250 \mathrm{~m}$ de altitud. Siete de estas especies se encuentran representadas dentro del Sistema Nacional de Áreas Naturales Protegidas por el Estado.

Palabras claves: Lamiaceae, Salvia, Perú, endemismo, plantas endémicas.

\section{Abstract}

The Lamiaceae are represented in Peru by around 21 genera and 190 species (Brako \& Zarucchi, 1993; Ulloa Ulloa et al., 2004), mainly herbs and shrubs. Here we recognize 57 endemic species in nine genera. Salvia is the genus with the largest number of endemic species. Endemic Lamiaceae species are found mostly in the Mesoandean and Very Humid Montane Forest regions, between 1500 and $4250 \mathrm{~m}$ elevation. Seven endemic species of Lamiaceae have been recorded to date in the Peruvian protected areas system.

Keywords: Lamiaceae, Salvia, Peru, endemism, endemic plants.

\section{Clinopodium argenteum (Kunth) Govaerts}

$$
\text { VU, Blab(iii) }
$$

Publicación: World Checklist of Seed Plants 3(1): 16. 1999.

Colección tipo: A. Bonpland s.n.

Herbanios: B, P.

Nombre común: D esconocido.

Registro departamental: CA, HU, JU, LI.

Regiones Ecológicas: MA; $1880 \mathrm{~m}$.

SINAN PE: Sin registro.

Herbarios peruanos: Ninguno.

Observaciones: Especie arbustiva descrita de una planta recolectada en 1802, en la cuenca del Llaucano. Esta especie es conocida también en la cuenca del Chamaya y Alto Marañón, extendiéndose su distribución hasta la parte central del país.

\section{Clinopodium clivorum (E pling) Harley \& A. Granda EN, Blab(iii)}

Publicación: World Checklist of Seed Plants 3: 16. 1999. Colección tipo: A. López M. 1555

Herbarios: F, K, LA, M, MICH, MO, NY, UC, UCLA, US; HUT!.

Nombre común: D esconocido

Registro departamental: CA, LL.

Regiones Ecológicas: MA, PAR; 2700-3150 m.

SINAN PE: Sin registro.

Herbarios penuanos: HUT (isotipo), USM (1).

Observaciones: Arbusto, conocido aparentemente sólo de dos localidades, en la vertiente del Pacífico. Esta especie habita laderas rocosas y no havuelto a ser recolectada desde la década de 1960. En Brako \& Zarucchi (1993) fue incluida en Saturga(Harley \& G randa Paucar, 2000).

\section{Clinopodium cylindrostachys (E pling \& Játiva) Govaerts}

$$
\text { EN, Blab(iii) }
$$

Publicación: World Checklist of Seed Plants 3(1): 17. 1999.

Colección tipo: P.C. Hutchison \& J.K. Wright 3869

Herbarios: F, K, LA, M, MICH, MO, NY, UC, UCLA, US; USM!.

Nombre común: Desconocido.

Registro departamental: AM.

Regiones Ecológicas: BPM, BMHM; $1700-2550 \mathrm{~m}$.

SINANPE: Sin registro.

Herbarios peruanos: USM (isotipo).

Observaciones: Especie herbácea restringida a Amazonas, de laderas en las cuencas del Alto Marañón y Utcubamba.

\section{Clinopodium flabellifolium (E pling \& Játiva) Govaerts

$$
\text { VU, B lab(iii) }
$$

Publicación: World Checklist of Seed Plants 3(1): 17. 1999.

Colección tipo: R. Ferreyra 13740

Herbarios: LA, MO.

Nombre común: D esconocido.

Registro departamental: CA, PI.

Regiones Ecológicas: MDE, MA; 1900-2200 m.

SINAN PE: Sin registro.

Herbarios peruanos: Ninguno.

Observaciones: Especie arbustiva, conocida aparentemente de dos localidades. La colección tipo proviene de la cuenca del Huancabamba. También es conocida de la cuenca del Jequetepeque.

Nota del Editor: En la versión on line de este artículo han sido omitidos los mapas del Perú que ilustraban el Registro departamental. Para ubicar las abreviaturas de los departamentos vea al final del artículo. 
5. Clinopodium junctionis (Epling \& Játiva) Govaerts

$$
\text { EN, Blab(iii) }
$$

Publicación: World Checklist of Seed Plants 3(1): 17. 1999.

Colección tipo: A. López M. \& A. Sagástegui A. 3533

Herbarios: LA; HUT!.

Nombre común: Desconocido.

Registro departamental: LL.

Regiones Ecológicas: MA; $3100 \mathrm{~m}$.

SINANPE: Sin registro.

Herbarios penuanos: HUT (isotipo).

Observaciones: Especie arbustiva, conocida solamente del material tipo, una planta recolectada en 1961, en unazona intermedia entre la región paramuna y la mesoandina en el norte del país, la cual requiere de mayor herborización.

\section{Clinopodium mathewsii (Briq.) Govaerts}

$$
\text { VU, Blab(iii) }
$$

Publicación: World Checklist of Seed Plants 3(1): 17. 1999.

Colección tipo: A. Mathews s.n.

Herbarios: F, G, K, M, MICH, MO, NY, P, UC, UCLA, US.

Nombre común: D esconocido.

Registro departamental: AM, CA.

Regiones Ecológicas: MA, BMHM; 1800- $2400 \mathrm{~m}$.

SINANPE: Sin registro.

Hemarios peruanos: MOL (1), USM (5).

Observaciones: Especie arbustiva, recolectada originalmente en el siglo XIX. Esta especie es conocida de relictos de bosque, monte bajo pedregoso y laderas rocosas de la cuencas del Chamaya y Utcubamba, al norte del país.

\section{Clinopodium plicatulum (Epling) Govaerts EN, Blab(iii)}

Publicación: World Checklist of Seed Plants 3(1): 17. 1999.

Colección tipo: J.F. Macbride \& W. Featherstone 2238

Herbarios: F, MO.

Nombre común: D esconocido.

Registro departamental: HU.

Regiones Ecológicas: MA; 2135 m.

SINANPE: Sin registro.

Herbarios peruanos: Ninguno.

Observaciones: Especie arbustiva, conocida solamente de la colección tipo, una planta recolectada en 1922, al oeste del Departamento de Huánuco, cuenca del Alto Marañón.

\section{Clinopodium pulchellum (Kunth) Govaerts}

\section{LC}

Publicación: World Checklist of Seed Plants 3(1): 18. 1999.

Colección tipo: A. Humboldt s.n.

Herbarios: B.

Nombre común: Panizara; limoncillo.

Registro departamental: AN, CA, JU, LL, PI. Regiones Ecológicas: MA, PAR, BS; 2800-3500 m.

SINAN PE: Sin registro.
Herbarios penuanos: MOL (2), USM (16).

Observaciones: Especie arbustiva o subarbustiva ampliamente distribuida en el centro y norte del país, en laderas rocosas yjalcas de las cuencas del Chicama, Alto Marañón, Crisneja y Santa.

\section{Clinopodium sericifolium (E pling \& Játiva) Govaerts}

$$
\text { EN, B1ab(iii) }
$$

Publicación: World Checklist of Seed Plants 3(1): 19. 1999.

Colección tipo: A. López M. \& A. Sagástegui A. 3189

Hemarios: UC; HUT!

Nombre común: Desconocido.

Registro departamental: LL.

Regiones Ecológicas: MA; 3200 m.

SINANPE: Sin registro.

Herbarios peruanos: CPUN (1), HUT (isotipo+1).

Observaciones: Especie arbustiva, conocida de laderas boscosas y matorrales de la cuenca del Alto Marañón, en el extremo norte de La Libertad.

\section{Clinopodium speciosum (H ook.) Govaerts}

\section{LC}

Publicación: World Checklist of Seed Plants 3(1): 19. 1999.

Colección tipo: A. Cruckshanks s.n.

Henbarios: $\mathrm{K}$.

Nombre común: Chipita, muña.

Registro departamental: AN, JU, LI.

Regiones Ecológicas: MA, PSH; 3200$4000 \mathrm{~m}$.

SINANPE: PNH

Herbarios peruanos: MOL (6), USM (35).

Observaciones: Especie arbustiva, descrita de una planta recolectada en la cuenca del Chillón en el siglo XIX. Ha sido recolectada en varios ambientes distintos como pajonales de puna, matorrales, laderas rocosas, campos de cultivo y bosques de Pdylepis O tras localidades con poblaciones de esta especie incluye el Parque Nacional Huascarán.

\section{Clinopodium sphenophyllum (Epling) Govaerts}

$$
\text { EN, Blab(iii) }
$$

Publicación: World Checklist of Seed Plants 3(1): 19. 1999.

Colección tipo: E.P. Killip \& A.C. Smith 24443

Herbarios: US.

Nombre común: D esconocido.

Registro departamental: JU.

Regiones Ecológicas: BPM, BMHM; 2700-3200 m.

SINAN PE: Sin registro.

Herbarios peruanos: USM (1).

Observaciones: Especie arbustiva, registrada solamente para la parte central de Junín, en borde de bosques de la cuenca del Perené. 


\section{Clinopodium striatum (Ruiz \& Pav.) Govaerts}

$$
\text { VU, B lab(iii) }
$$

Publicación: World Checklist of Seed Plants 3(1): 19. 1999

Colección tipo: H. Ruiz \& J. Pavón s.n.

Herbarios: MA.

Nombre común: D esconocido.

Registro departamental: HU.

Regiones Ecológicas: BPM; 2700-3300

m.

SINANPE: Sin registro.

Herbarios peruanos: MOL (1), USM (3).

Observaciones: Especie arbustiva, registrada para bosques montanos y montes perennifolios en la cuenca del Alto Huallaga, en la parte central de Huánuco.

\section{Clinopodium vargasii (E pling \& Mathias) Govaerts}

$$
\text { EN, B 1ab(iii) }
$$

Publicación: World Checklist of Seed Plants 3(1): 19. 1999.

Colección tipo: C. Vargas C. 9472

Herbarios: LA.

Nombre común: D esconocido.

Registro departamental: CU.

Regiones Ecológicas: BMHM; 2600$3200 \mathrm{~m}$.

SINAN PE: PNM

Herbarios peruanos: MOL( 1), USM (1)

Observaciones: Arbusto conocido del sur del país. El ejemplar tipo fue recolectado en 1950, en la cuenca alta del Alto Madre de Dios, en un área ubicada hoy en el Parque Nacional Manu. Una colección adicional proviene de la cuenca del Yavero.

\section{Clinopodium weberbauen (Mansf.) Govaerts}

\section{NT}

Publicación: World Checklist of Seed Plants 3(1): 19. 1999.

Colección tipo: A. Weberbauer 7016

Herbarios: B; MOL!

Nombre común: O régano cangle.

Registro departamental: AN, CA, LL.

Regiones Ecológicas: MA, PSH, PAR; 2580- $4000 \mathrm{~m}$.

SINAN PE: PNH, SNC

Herbarios peruanos: HUT (16), MOL (isotipo+2), USM (4).

Observaciones: Especie arbustiva, recolectada en matorrales y laderas rocosas, en las cuencas del Santa y Alto Marañón.

\section{Hyptis elongata Benth.}

\section{VU, B lab(iii)}

Publicación: Labiat. Gen. Spec. 88. 1833. Colección tipo: H. Ruiz \& J. Pavón s.n. Herbarios: B.

Nombre común: D esconocido.

Registro departamental: LI.

Regiones Ecológicas: D ST, MDE, MA; 800- $2200 \mathrm{~m}$.

SINAN PE: Sin registro.

Hembarios peruanos: USM (7).
Observaciones: Especie subarbustiva, conocida de varias localidades en la vertiente occidental y restringida al D epartamento de Lima. Esta especie crece en laderas rocosas y cauces secos, en las cuencas del Rímac y Chillón.

\section{Hyptis mamubifolia Epling \& Mathias}

EN, Blab(iii)

Publicación: Brittonia 8: 299. 1957.

Colección tipo: E.L. Evinger 508

Herbarios: US.

Nombre común: D esconocido.

Registro departamental: AM.

Regiones Ecológicas: MA; 2320 m.

SINAN PE: Sin registro.

Hembarios peruanos: Ninguno.

Observaciones: Hierba conocida solamente de la descripción original, una planta recolectada en 1943 al sur de Amazonas, creciendo sobre arenisca. Estalocalidad corresponde ala cuenca del Alto Marañón y el tipo de ambiente ha sido poco herborizado.

\section{Lepechinia marica E pling \& Mathias \\ CR, Bla}

Publicación: Brittonia 8(4): 305. 1957.

Colección tipo: A. López M. 601

Herbarios: US; HUT!

Nombre común: D esconocido.

Registro departamental: LL.

Regiones Ecológicas: MA; $2900 \mathrm{~m}$.

SINAN PE: Sin registro.

Herbarios penuanos: HUT (isotipo).

Observaciones: Arbusto aparentemente conocido solamente de la colección original, una planta recolectada en 1951, en el noroccidente de La Libertad, en la cuenca del Chicama.

\section{Lepechinia mollis Epling}

\section{LC}

Publicación: Repert. Spec. Nov. Regni Veg. Beih. 85: 27. 1935.

Colección tipo: A. Weberbauer 7106

Henbarios: $F$.

Nombre común: D esconocido.

Registro departamental: CA, LA, LL, PI. Regiones Ecológicas: MDE, MA; 1750$2680 \mathrm{~m}$.

SINAN PE: Sin registro.

Herbarios peruanos: HAO (1), HUT (1)?, USM (1)?.

Observaciones: Especie arbustiva conocida de varias localidades en el norte del país. La mayoría de los ejemplares provienen de las cuencas del Olmos, Crisneja, Chicamay Jequetepeque. Esta especie es conocida además del valle internadino de Huancabamba.

\section{Lepechinia scobina Epling}

\section{NT}

Publicación: Repert. Spec. Nov. Regni Veg. Beih. 85: 26. 1935.

Colección tipo: C. Jelski 73

Hemarios: W.

Nombre común: D esconocido.

Registro departamental: CA, LL, PI.

Regiones Ecológicas: MA; 2500-3500 m.

SINAN PE: Sin registro.

Hemanios peruanos: CPUN (2). 
Observaciones: Arbusto, conocido del norte del país, de poblaciones densas en las cuencas del Chicama y Crisneja. Habita ambientes intervenidos y matorrales.

\section{Lepechinia tomentosa (Benth.) Epling}

$$
\text { EN, Blab(iii) }
$$

Publicación: Repert. Spec. Nov. Regni Veg. Beih. 85: 19. 1935.

Colección tipo: J. D ombey 278

Herbarios: GH.

Nombre común: D esconocido.

Registro departamental: LI.

Regiones Ecológicas: MA, PSH; 3500$3540 \mathrm{~m}$.

SINANPE: Sin registro.

Herbarios peruanos: USM (1).

Observaciones: Especie herbácea, conocida de matorrales y pajonales en la región central del país. Esta especie fue descrita de una planta recolectada en la cuenca del Checras en el siglo XIX. Una localidad adicional se conoce de la cuenca del Chillón.

\section{Mesosphaerum salvioides Zahlbr.}

\section{DD}

Publicación: Ann. K.K. Naturhist. Hofmus. 7(1-2): 9-10. 1892.

Colección tipo: C. Jelski 73

Herbarios: W.

Nombre común: D esconocido.

Registro departamental: CA.

Regiones Ecológicas: Sin datos; altitud desconocida.

SINANPE: Sin registro.

Herbarios peruanos: Ninguno.

Observaciones: Especie subarbustiva, conocida solamente de la descripción original, una planta recolectada en el siglo XIX sin datos de localidad y hábitat precisos.

\section{Minthostachys salicifolia Epling}

$$
\text { EN, Blab(iii) }
$$

Publicación: Repert. Spec. Nov. Regni Veg. Beih. 85: 165. 1936.

Colección tipo: A. Weberbauer 7578

Herbarios: $F$.

Nombre común: D esconocido.

Registro departamental: AY.

Regiones Ecológicas: BMHM; 2900$3000 \mathrm{~m}$.

SINANPE: Sin registro.

Herbarios peruanos: Ninguno.

Observaciones: Especie subarbustiva, conocida solamente de la colección original, una planta recolectada en 1926, en el valle de Choimacota, cuenca del Apurímac. Esta zona ha sido escasamente herborizada aún cuando alberga numerosos endemismos.

\section{Salvia atrocalyx Epling}

\section{CR, Blab(iii)}

Publicación: Repert. Spec. Nov. Regni Veg. Beih. 85: 65. 1935.

Colección tipo: E.P. Killip \& A.C. Smith 24401

Hemarios: UC, US.

Nombre común: D esconocido.

Registro departamental: JU.

Regiones Ecológicas: BMHM; 2700$3200 \mathrm{~m}$.

SINAN PE: Sin registro.

Herbarios peruanos: Ninguno.

Observaciones: Especie herbácea, conocida solamente de la colección tipo, una planta recolectada en 1929 en los bosques de la cuenca del Palca, afluente del Tulumayo. Los ambientes naturales de estas cuencas han sido extensamente modificados por la expansión agnícola.

\section{Salvia ayavacensis Kunth}

\section{EN, B 1ab(iii)}

Publicación: Nov. Gen. Sp. 2: 298. 1818. Colección tipo: A. Humboldt \& A. Bonpland s.n.

Henbarios: $P$.

Nombre común: D esconocido.

Registro departamental: PI.

Regiones Ecológicas: MA, BS; 2100$2900 \mathrm{~m}$.

SINANPE: Sin registro.

Herbarios penuanos: HAO (1), HUT (1), USM (1).

Observaciones: Especie subarbustiva, conocida solamente para el norte del D epartamento de Piura, en bosques y matorrales de las cuencas del Chamaya y Chira. Esta especie fue originalmente recolectada a inicios del siglo XIX.

\section{Salvia bullulata Benth.}

\section{EN, Bla}

Publicación: Prodr. 12: 327. 1848.

Colección tipo: A. Mathews 2132

Herbarios: BM, K, US.

Nombre común: D esconocido.

Registro departamental: AM, LA.

Regiones Ecológicas: BS; $1975 \mathrm{~m}$.

SINANPE: Sin registro.

Herbarios penuanos: HUT (1).

Observaciones: Arbusto, conocido de dos localidades en el norte del país. Esta especie no fue listada como endémica por Brako \& Zarucchi (1993). A parentemente no ha vuelto a ser recolectada desde 1963.

\section{Salvia camponum Epling}

$$
\text { EN, B lab(iii) }
$$

Publicación: Bull. Torrey Bot. Club 71(5): 489-490. 1944.

Colección tipo: R. Metcalf 30805

Herbarios: MO, US.

Nombre común: D esconocido.

Registro departamental: AM.

Regiones Ecológicas: MA; 2350 - 2500 m.

SINANPE: Sin registro.

Herbarios penuanos: USM (1). 
Observaciones: Especie herbácea, conocida solamente del sur de Amazonas, en pajonales semixéricos de la cuenca del Utcubamba. Esta especie fue recolectada inicialmente en 1942.

\section{Salvia consobrina E pling}

\section{LC}

Publicación: Brittonia 12(2): 149. 1960.

Colección tipo: A. López M. et al. 2281

Herbarios: LA; HUT!.

Nombre común: D esconocido.

Registro departamental: CA, LL, LA, PI, TU.

Regiones Ecológicas: MDE; 800-1700

m.

SINAN PE: Sin registro.

Herbarios peruanos: HAO (1), HUT

(isotipo+1), USM (3), MOL(1).

Observaciones: Especie arbustiva o bejuco, bien representada en la región norte del país. Esta especie es conocida solamente para la vertiente del Pacífico, creciendo en bordes de camino, laderas y matorrales de las cuencas del Bocapán, Chira, Jequetepeque, PiuraCascajal y Olmos.

\section{Salvia cnuikshanksii Benth.}

\section{LC}

Publicación: Labiat. Gen. Spec. 261. 1833.

Colección tipo: A. Mathews 462

Herbarios: G.

Nombre común: D esconocido.

Registro departamental: AN, CA, LL, LA, LI.

Regiones Ecológicas: MA; 2200-3500 $\mathrm{m}$.

SINAN PE: Sin registro.

Herbarios penuanos: HUT (1), MOL (1), USM (17).

Observaciones: Especie arbustiva, conocida de la vertiente del Pacífico. La colección tipo fue recolectada en el siglo XIX en la cuenca del Chillón. Ha sido recolectada en varias localidades con vegetación de matorrales y laderas rocosas en la región centro-norte del país.

\section{Salvia cuspidata Ruiz \& Pav.}

\section{NT}

Publicación: Fl. Peruv. 1: 23, t. 40. 1798. Colección tipo: J. D ombey s.n.

Herbarios: MA.

Nombre común: D esconocido.

Registro departamental: AN, CA, HU, LI. Regiones Ecológicas: MA, BS; 1800$3300 \mathrm{~m}$.

SINAN PE: Sin registro.

Herbarios penuanos: CPUN (2), HUT (1), MOL (6), USM (10).

Observaciones: Especie arbustiva, descrita de una planta recolectada en el siglo XVIII, de la cuenca alta del Checras, una zona escasamente herborizada. Otras poblaciones de esta especie han sido muestreadas en matorrales y laderas pedregosas de las cuencas del Rímac, Marañón y Santa.

\section{Salvia cyanicalyx Epling}

\section{LC}

Publicación: Bull. Torrey Bot. Club 68: 564. 1941.

Colección tipo: H.E. Stork et al. 10618

Hembarios: F.

Nombre común: D esconocido.

Registro departamental: AP, AY.

Regiones Ecológicas: BMHM; 2800$3100 \mathrm{~m}$.

SINAN PE: SNAM

Herbarios peruanos: USM (4).

Observaciones: Especie herbácea, registrada para la zona central del país, en matorrales y bosques montanos de las cuencas del Apurímac, Mantaro y Pampas. El ejemplar tipo fue recolectado en 1939 en lo que hoy es el Santuario Nacional de Ampay.

\section{Salvia cylindriflora E pling}

\section{DD}

Publicación: Repert. Spec. Nov. Regni Veg. Beih. 85: 122. 1935.

Colección tipo: R.W. Pearce s.n.

Hemarios: K, LA.

Nombre común: D esconocido.

Registro departamental: PI.

Regiones Ecológicas: Sin datos; altitud desconocida.

SINAN PE: Sin registro.

Herbarios peruanos: Ninguno.

Observaciones: Especie arbustiva, conocida solamente del material tipo, unaplanta recolectadaamediados del siglo XIX, en el nortedel país, probablemente en bosques de la cuenca del Chamaya. Aparentemente no havuelto a ser recolectada desde entonces, al igual que otras especies de ambientes semixéricos en valles interandinos.

\section{Salvia grisea E pling \& Mathias}

\section{NT}

Publicación: Brittonia 8(4): 308. 1957.

Colección tipo: R. Ferreyra 7628

Herbarios: UC, US; MOL!, USM!.

Nombre común: Agaruro.

Registro departamental: AN, LA, LL.

Regiones Ecológicas: MA, PAR; 2750$3500 \mathrm{~m}$.

SINANPE: Sin registro.

Herbanios penuanos: HAO (2), HUT (3), MOL (isotipo), USM (isotipo).

Observaciones: Especie subarbustiva, conocida en la vertiente occidental y valles interandinos del norte del país. Esta especie habita laderas rocosas y bordes de carretera, en las cuencas del Chicama, Alto Marañón y Santa.

\section{Salvia griseifolia E pling}

\section{DD}

Publicación: Repert. Spec. Nov. Regni Veg. Beih. 85: 78. 1935.

Colección tipo: W. Lobb 237

Herbarios: $\mathrm{K}$.

Nombre común: D esconocido.

Registro departamental: HU.

Regiones Ecológicas: Sin datos; altitud desconocida.

SINANPE: Sin registro.

Herbarios peruanos: Ninguno. 
Observaciones: Arbusto, conocido aparentemente sólo de dos ejemplares de herbario recolectados en el siglo XIX, sin datos de procedencia exactos.

\section{Salvia hapalophylla Epling}

$$
\text { EN, Blab(iii) }
$$

Publicación: Repert. Spec. Nov. Regni Veg. Beih. 85: 122. 1936.

Colección tipo: A. Weberbauer 7858

Herbarios: F, UC.

Nombre común: D esconocido.

Registro departamental: CU, PU.

Regiones Ecológicas: MA; 1800-2300 m.

SINANPE: Sin registro.

Herbarios peruanos: USM (1).

Observaciones: Arbusto, conocido solamente de dos localidades en el sur del país. La colección tipo fue recolectada en 1929, del valle de Marcapata, en la cuenca del Inambari. Los ambientes naturales en este valle están reducidos debido a la deforestación e incendios intencionales asociados con agricultura. Si bien en este valle han sido registrados varios endemismos como esta especie, no hay un estudio florístico ni tampoco una evaluación de campo para ubicar y evaluar poblaciones de interés a la conservación.

\section{Salvia incurvata Ruiz \& Pav.}

\section{CR, Blab(iii)}

Publicación: Fl. Peruv. 1: 24, t. 42, f. b. 1798.

Colección tipo: H. Ruiz \& J. Pavón s.n.

Herbarios: B.

Nombre común: D esconocido.

Registro departamental: HU.

Regiones Ecológicas: BMHM; altitud desconocida.

SINAN PE: Sin registro.

Herbarios peruanos: Ninguno.

Observaciones: Especie herbácea, conocida sólo de la colección tipo, una planta recolectada en el siglo XVIII, probablemente en bosques montanos hoy desaparecidos, en la cuenca del Pachitea.

\section{Salvia innoxia E pling}

\section{CR, B1ab(iii)}

Publicación: Brittonia 8(4): 309. 1957.

Colección tipo: R. Ferreyra 7555

Herbarios: UC, US; MOL!, USM!

Nombre común: Desconocido.

Registro departamental: AN.

Regiones Ecológicas: MA; $3000 \mathrm{~m}$.

SINAN PE: Sin registro.

Herbarios penuanos: MOL (isotipo), USM (isotipo).

Observaciones: Subarbusto, conocido solamente del material original, una planta recolectada en 1950, en la cuenca alta del Pativilca, una zona que requiere mayor información florística. Por habitar ambientes pedregososy semixéricos tal vez pase desapercibida. Sus poblaciones podrían estar afectadas por los incendios intencionales.

\section{Salvia lachnostoma E pling}

EN, B 1ab(iii)

Publicación: Repert. Spec. Nov. Regni Veg. Beih. 85: 117. 1936.

Colección tipo: J.F. Macbride 4478

Hemarios: F, MO.

Nombre común: D esconocido.

Registro departamental: HU.

Regiones Ecológicas: BMHM; 2440$2475 \mathrm{~m}$.

SINAN PE: Sin registro.

Hembarios peruanos: Ninguno.

Observaciones: Arbusto decumbente, conocido aparentemente de dos localidades en el centro del país, ambas en la cuenca del Huallaga. Esta especie fue recolectada tanto en el siglo XIX y posteriormente en 1923. Las localidades conocidas en la Provincia de Pachitea albergan otros endemismos, pero han sido escasamente herborizadas. Incendios intencionales y deforestación podrían ser de preocupación.

\section{Salvia lanicaulis E pling \& Játiva}

\section{LC}

Publicación: Brittonia 15(4): 374. 1963. Colección tipo: A. López M. \& A. Sagástegui A. HUT-1422

Hembarios: LA; HUT!

Nombre común: Desconocido.

Registro departamental: CA, LL.

Regiones Ecológicas: MA, PAR; 3300$4000 \mathrm{~m}$.

SINANPE: Sin registro.

Herbarios penuanos: CPUN (1), HAO (2), HUT (isotipo+6).

Observaciones: Especie arbustiva, conocida de varias localidades en la región mesoandina y paramuna del norte del país. Esta especie habita matorrales en ladera rocosas y suelos calizos, en las cuencas del Alto Marañón, Crisnejay Llaucano. Localmente sus poblaciones son densas.

\section{Salvia medusa Epling \& Játiva}

\section{CR, Blab(iii)}

Publicación: Brittonia 15(4): 373. 1963. Colección tipo: A. López M. \& A. Sagástegui A. 3535

Hemanios: UC; HUT!

Nombre común: Desconocido.

Registro departamental: LL.

Regiones Ecológicas: PAR; $3100 \mathrm{~m}$.

SINAN PE: Sin registro.

Hembarios penuanos: HUT (isotipo+1).

Observaciones: Subarbusto conocido solamente del material tipo, una planta recolectada en ambientes paramunos, de una subcuenca del Marañón. Aparentemente no ha vuelto a ser recolectada desde 1961. Amenazas a sus poblaciones podrían estar asociadas a actividades mineras e incendios intencionales con fines agrícolas. 


\section{Salvia obumbrata Epling}

$$
\text { EN, Bla }
$$

Publicación: Repert. Spec. Nov. Regni Veg. Beih. 105: 39. 1938.

Colección tipo: E.P. Killip \& A.C. Smith 25311

Hembarios: $F, K$.

Nombre común: D esconocido.

Registro departamental: JU, SM.

Regiones Ecológicas: BMHP; altitud desconocida.

SINAN PE: Sin registro.

Herbarios peruanos: Ninguno.

Observaciones: Especie herbácea o subarbustiva, conocida de la colección tipo, una planta recolectada en la cuenca del Perené, en 1929. Macbride (1960) incluyó con duda un ejemplar de Richard Spruce recolectado en el siglo XIX en la cuenca del Mayo. Para ambas localidades no existen recolecciones recientes de esta especie.

\section{Salvia pavonii Benth.}

\section{EN, Blab(iii)}

Publicación: Labiat. Gen. Spec. 278. 1833.

Colección tipo: H. Ruiz \& J. Pavón s.n.

Herbarios: MA.

Nombre común: D esconocido.

Registro departamental: JU.

Regiones Ecológicas: BMHM; $2900 \mathrm{~m}$.

SINAN PE: Sin registro.

Hemarios peruanos: Ninguno.

Observaciones: Especie herbácea o subarbustiva, conocida solamente del centro del país, en bosques montanos y matorrales, probablemente, extintos de la cuenca del Perené. Esta especie no ha vuelto, al parecer, a ser recolectada desde la década de 1950.

\section{Salvia perlucida Epling}

\section{CR, B 1ab(iii)}

Publicación: Repert. Spec. Nov. Regni Veg. Beih. 85: 72. 1935.

Colección tipo: E.P. Killip \& A.C. Smith 22350

Henbarios: MO, NY, UC, US.

Nombre común: D esconocido.

Registro departamental: AY.

Regiones Ecológicas: BMHM; 1500 m.

SINAN PE: Sin registro.

Hembarios peruanos: Ninguno.

Observaciones: Especie arbustiva, conocida sólo del material tipo, una planta recolectada en 1929, de un valle boscoso de la cuenca del Apurímac, al suroeste de los límites del Parque Nacional Otishi. Amenazas a sus poblaciones podrían estar asociadas a las derivadas de las actividades agrícolas.

\section{Salvia plumosa Ruiz \& Pav.}

\section{EN, Blab(iii)}

Publicación: Fl. Peruv. 1: 26, t. 37. 1798.

Colección tipo: J. D ombey 270

Herbarios: MA.

Nombre común: Chenchelcoma.

Registro departamental: HU, JU.

Regiones Ecológicas: MA; altitud desconocida.

SINANPE: Sin registro.

Herbarios peruanos: Ninguno.
Observaciones: Arbusto conocido sólo para el centro del país, en las cuencas del Perené y Huallaga. Esta especie está representada por colecciones realizadas hasta la década de 1960. Amenazas a sus poblaciones, en fragmentos de bosques, sea la deforestación.

\section{Salvia pseudorosmaninus Epling}

$$
\text { EN, Blab(iii) }
$$

Publicación: Bull. Torrey Bot. Club 68: 557. 1941.

Colección tipo: H.E. Stork \& O.B. Horton 9968

Herbarios: UC.

Nombre común: D esconocido.

Registro departamental: LL.

Regiones Ecológicas: MA; 2800-3250 $\mathrm{m}$.

SINAN PE: Sin registro.

Henbarios penuanos: HAO (1), HUT (2), USM (1).

Observaciones: Especie arbustiva, conocida solamente de una localidad en la cuenca del Tablachaca, un afluente del Santa, donde ha sido recolectada en diferentes ocasiones, siendo su rango altitudinal menor a los $500 \mathrm{~m}$. Crece en laderas arbustivas, que podrían estar afectadas por incendios intencionales.

\section{Salvia psilantha E pling}

$$
\text { CR, Blab(iii) }
$$

Publicación: Repert. Spec. Nov. Regni Veg. Beih. 85: 121. 1936.

Colección tipo: A. Weberbauer 6590

Hemanios: F, LA, US; MOL!.

Nombre común: D esconocido.

Registro departamental: JU.

Regiones Ecológicas: MA; 2300-2400

m.

SINAN PE: Sin registro.

Herbarios peruanos: MOL (isotipo).

Observaciones: Especie herbácea conocida solamente de la colección tipo, una planta recolectada en 1913, en el valle del Pariahuanca, un afluente del Mantaro, en la parte sur de Junín. Este sector de Junín, que parece formar parte de la zona que Weberbauer (1945) describió como la Cordillera de Huaritanga, no ha recibido mayores estudios botánicos. En esta parte del Mantaro, concurren bosques perennifolios con matorrales extensos.

\section{Salvia sarmentosa E pling}

\section{NT}

Publicación: Repert. Spec. Nov. Regni Veg. Beih. 85: 79. 1935.

Colección tipo: F.W. Pennell 13650

Herbarios: F, GH, NY, S, UC, US.

Nombre común: D esconocido.

Registro departamental: AP, CU.

Regiones Ecológicas: MA, PSH; 3000$4200 \mathrm{~m}$.

SINAN PE: Sin registro.

Herbarios penuanos: CUZ (3)?, HUT (1), USM (4).

Observaciones: Especie herbácea 0 arbustiva conocida solamente del sur del país, donde habita matorrales y laderas secas, en las cuencas del Mantaro y Urubamba. Ha sido recolectada en varias oportunidades de los alrededores de la localidad tipo, en la cuenca del Urubamba. 


\section{Salvia silvarum E pling}

CR, Blab(iii)

Publicación: Repert. Spec. Nov. Regni Veg. Beih. 85: 91. 1935.

Colección tipo: E.P. Killip \& A.C. Smith 25612

Herbarios: LA, NY, US.

Nombre común: D esconocido.

Registro departamental: PA.

Regiones Ecológicas: BMHP; 900- 1600

m.

SINAN PE: Sin registro.

Herbarios peruanos: Ninguno.

Observaciones: Arbusto, conocido sólo del material tipo, una planta recolectada en parte de la trocha que unía el valle de Chanchamayo con la cuenca del Cacazú. Al parecer, no ha vuelto a ser recolectada desde 1929. Podría estar representada en el Parque Nacional Yanachaga-Chemillén.

\section{Salvia striata Benth.}

\section{LC}

Publicación: Prodr. 12: 343. 1848.

Colección tipo: A. Mathews 3155

Herbarios: $G, K$.

Nombre común: D esconocido.

Registro departamental: AM, AP, AR, CA, CU, LL.

Regiones Ecológicas: MA, BPM, BMHM; 1860- $3700 \mathrm{~m}$.

SINANPE: Sin registro.

Hembarios penuanos: HUT (4), USM (16).

Observaciones: Hierba o subarbusto, de amplio rango en distribución, desde Cajamarca hasta Arequipa, en laderas rocosas y bosque caducifolio interandino, en las cuencas del Apurímac, Alto Marañón, Mantaro, Moche, Utcubamba y Urubamba.

\section{Salvia subscandens E pling \& Játiva}

\section{CR, Blab(iii)}

Publicación: Brittonia 20(4): 308. 1968. Colección tipo: P.C. Hutchison \& J.K. Wright 3421

Hemarios: LA, MO, UC; USM!.

Nombre común: D esconocido.

Registro departamental: LA.

Regiones Ecológicas: MDE; 1150- 1200

$\mathrm{m}$.

SINAN PE: Sin registro.

Herbarios penuanos: USM (isotipo).

Observaciones: Especie herbácea o subarbustiva, conocida solamente del ejemplar tipo, una planta recolectada en 1964, en la cuenca del Motupe-La Leche-Chancay. Aparentemente no havuelto a ser recolectada desde entonces, si bien esta planta crece en una de las rutas más transitadas de acceso al nor-oriente del país.

\section{Salvia tubulosa E pling}

$$
\text { EN, Blab(iii) }
$$

Publicación: Repert. Spec. Nov. Regni Veg. Beih. 85: 115-116. 1936.

Colección tipo: R.W. Pearce 261

Herbarios: $\mathrm{K}$.

Nombre común: D esconocido.

Registro departamental: PA.

Regiones Ecológicas: BMHP; 1870 m.

SINANPE: Sin registro.

Herbarios penuanos: AMAZ (1).

Observaciones: Especie arbustiva, conocida aparentemente de dos localidades, ambas en el oriente del Departamento de Pasco, donde habita bosques montanos. La localidad original estálocalizada en la cuenca del Pozuzo, en donde fue recolectada en el siglo XIX. En 1982 fue recolectada de la cuenca del Yamaquizú, un tributario del Iscozacín.

\section{Salvia vargasii E pling}

\section{CR, Blab(iii)}

Publicación: Bull. Torrey Bot. Club 74(6): 514. 1947.

Colección tipo: C. Vargas C. 5834

Herbanios: LA.

Nombre común: D esconocido.

Registro departamental: AP.

Regiones Ecológicas: MA; $2300 \mathrm{~m}$.

SINANPE: Sin registro.

Herbarios peruanos: Ninguno.

Observaciones: Arbusto conocido solamente del material tipo, una planta recolectada en 1946, en la cuenca del Apurímac, en el Departamento de Apurímac y no en Cusco, como fue citado por Epling (1947). Esta localidad es caracterizada por sus ambientes xéricos. A parentementeno havuelto a ser recolectada desde entonces.

\section{Salvia weberbaueni Epling}

$$
\text { CR, Blab(iii) }
$$

Publicación: Repert. Spec. Nov. Regni Veg. Beih. 85: 117. 1936.

Colección tipo: A. Weberbauer 6638

Herbarios: F, US; MOL!.

Nombre común: D esconocido.

Registro departamental: JU.

Regiones Ecológicas: BPM; 3200-3300

$\mathrm{m}$.

SINAN PE: Sin registro.

Henbarios penuanos: MOL (isotipo).

Observaciones: Especie arbustiva conocida solamente del material tipo, una planta recolectada en 1913, en bosques perennifolios, de la cuenca del Masamerich, un afluente del Perené. Weberbauer (1945) describió esta localidad, la cual aparentemente no ha vuelto a ser herborizada. Probablemente la deforestación sea una amenaza para el hábitat de esta especie. 


\section{Salvia xanthophylla E pling \& Játiva}

$$
\text { EN, B1ab(iii) }
$$

Publicación: Brittonia 15(4): 374. 1963. Colección tipo: A. López M. \& A. Sagástegui A. 1410

Herbarios: LA; HUT!, USM!.

Nombre común: D esconocido.

Registro departamental: LL.

Regiones Ecológicas: PAR; $3580-4000$

m.

SINANPE: Sin registro.

Herbarios peruanos: HAO (1), HUT

(isotipo+2), USM (isotipo+1).

Observaciones: Especie subarbustiva, registrada solamente del norte del país, en dos localidades que probablemente representen una sola población, en la cuenca del Crisneja. Esta especie fue recolectada de ambientes paramunos rocosos; tal vez por ello resiste incendios ocasionales.

\section{Scutellaria benthamiana (Mansf.) E pling}

\section{VU, B lab(iii)}

Publicación: Repert. Spec. Nov. Regni Veg. Beih. 85: 179. 1936.

Colección tipo: A. Weberbauer 6164

Herbarios: B.

Nombre común: Purun orégano.

Registro departamental: $\mathrm{AM}, \mathrm{AY}, \mathrm{CA}, \mathrm{CU}$.

Regiones Ecológicas: MA, PSH, BS; 1150- $3390 \mathrm{~m}$.

SINANPE: Sin registro.

Hembarios penuanos: MOL (3), USM (2).

Observaciones: Especie arbustiva, al parecer de amplia distribución, desde las jalcas del norte hasta valles interandinos de Cajamarca a Cusco.

\section{Scutellaria gardoquioides Benth.}

\section{NT}

Publicación: Prodr. 12: 430. 1848.

Colección tipo: A. Mathews s.n.

Herbarios: $\mathrm{K}$.

Nombre común: D esconocido.

Registro departamental: AM, AN, AP, AY, CA, CU, LL.

Regiones Ecológicas: MDE, MA, PAR; 500-3900 m.

SINANPE: PNC, PNH

Herbarios penuanos: CPUN (2), HAO (2), USM (8).

Observaciones: Arbusto, conocido de varias localidades interandinas desde Amazonas hasta Cusco. Esta especie habita matorrales, pajonales y laderas rocosas. Ha sido recolectada en por lo menos dos áreas protegidas.

\section{Sphacele codon (Epling) J.F. Macbride}

$$
\text { EN, B1a }
$$

Publicación: Field Mus. Nat. Hist., Bot. Ser. 13(5/ 2): 764. 1960.

Colección tipo: O.F. Cook \& G.B. Gilbert 1144

Herbarios: US

Nombre común: D esconocido.

Registro departamental: CU.

Regiones Ecológicas: BMHM; 1800 m.

SINANPE: SHMP

Herbarios peruanos: USM (1).

Observaciones: Arbusto, conocido solamente del sur del país. Habita matorrales semixéricos en el valle del Urubamba.

\section{Stachys peruviana D ombey ex Benth.}

\section{LC}

Publicación: Labiat. G en. Spec. 551. 1834.

Colección tipo: J. D ombey s.n.

Herbarios: G.

Nombre común: D esconocido.

Registro departamental: AN, AP, AY, CU, LL.

Regiones Ecológicas: PSH, PAR; 3300$4250 \mathrm{~m}$.

SINANPE: PNH, SNA

Herbarios peruanos: CPUN (2).

Observaciones: Hierba perenne, conocida de numerosas localidades, en un amplio rango altitudinal y ambiental. La colección tipo aparentemente fue recolectada cerca de la ciudad de Lima, en el siglo XVIII. 
Mapa del Perú indicando las abreviaturas de los departamentos

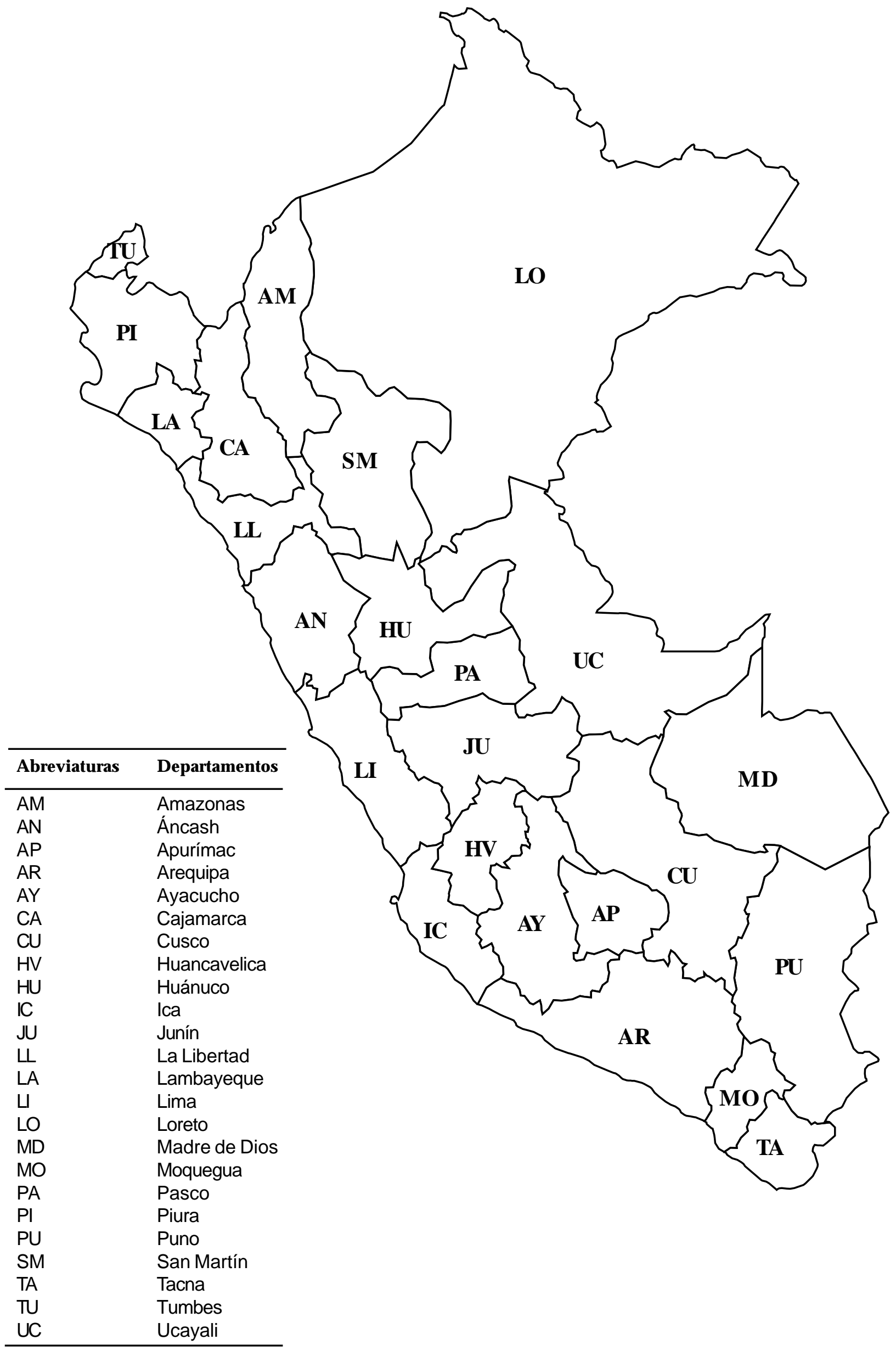

